Article

\title{
Oral Delivery of Probiotics Expressing Dendritic Cell-Targeting Peptide Fused with Porcine Epidemic Diarrhea Virus COE Antigen: A Promising Vaccine Strategy against PEDV
}

\author{
Xiaona Wang ${ }^{1, \dagger}{ }^{\dagger}$ Li Wang ${ }^{1,+}$, Xuewei Huang ${ }^{1}$, Sunting Ma ${ }^{1}$, Meiling Yu ${ }^{1}$, Wen Shi ${ }^{2}$, \\ Xinyuan Qiao ${ }^{1,3}$, Lijie Tang ${ }^{1,3}$, Yigang $X u^{1,3, *}$ and Yijing $\operatorname{Li}^{1,3, *}$ \\ 1 College of Veterinary Medicine, Northeast Agricultural University, Harbin 150030, China; \\ xiaonawang0319@163.com (X.W.); wanglicau@163.com (L.W.); huangxuewei126@126.com (X.H.); \\ masunting@163.com (S.M.); yu19890130@126.com (M.Y.); qiaoxinyuan@126.com (X.Q.); \\ tanglijie@neau.edu.cn (L.T.) \\ 2 College of Animal Science and Technology, Northeast Agricultural University, Harbin 150030, China; \\ wenshi_china@163.com \\ 3 Heilongjiang Key Laboratory for Animal Disease Control and Pharmaceutical Development, \\ Harbin 150030, China \\ * Correspondence: yigangxu_china@sohu.com (Y.X.); yijingli@163.com (Y.L.); Tel./Fax: +86-451-5519-0363 (Y.L.) \\ + These authors contributed equally to this work.
}

Received: 22 September 2017; Accepted: 23 October 2017; Published: 25 October 2017

\begin{abstract}
Porcine epidemic diarrhea virus (PEDV), an enteric coronavirus, is the causative agent of porcine epidemic diarrhea (PED) that damages intestinal epithelial cells and results in severe diarrhea and dehydration in neonatal suckling pigs with up to $100 \%$ mortality. The oral vaccine route is reported as a promising approach for inducing protective immunity against PEDV invasion. Furthermore, dendritic cells (DCs), professional antigen-presenting cells, link humoral and cellular immune responses for homeostasis of the intestinal immune environment. In this study, in order to explore an efficient oral vaccine against PEDV infection, a mucosal DC-targeting oral vaccine was developed using Lactobacillus casei to deliver the DC-targeting peptide (DCpep) fused with the PEDV core neutralizing epitope (COE) antigen. This probiotic vaccine could efficiently elicit secretory immunoglobulin A (SIgA)-based mucosal and immunoglobulin G (IgG)-based humoral immune responses via oral vaccination in vivo. Significant differences $(p<0.05)$ in the immune response levels were observed between probiotics expressing the COE-DCpep fusion protein and COE antigen alone, suggesting better immune efficiency of the probiotics vaccine expressing the DC-targeting peptide fused with PEDV COE antigen. This mucosal DC-targeting oral vaccine delivery effectively enhances vaccine antigen delivery efficiency, providing a useful strategy to induce efficient immune responses against PEDV infection.
\end{abstract}

Keywords: Lactobacillus; PEDV COE antigen; dendritic cell-targeting peptide; oral vaccine

\section{Introduction}

Porcine epidemic diarrhea virus (PEDV) infection, causing an acute and highly contagious enteric disease in nursing pigs with up to $100 \%$ mortality, mainly results in intestinal-epithelial-cell damage [1]. Currently, an RNA vaccine and an inactivated whole-virus vaccine of porcine epidemic diarrhea were developed at Harrisvaccines ${ }^{\mathrm{TM}}$ and Zoetis (Florham Park, NJ, USA), respectively, widely used and considered to be effective [2]. PEDV mainly gains entry to the host via the intestinemucosal surfaces of the piglets. Therefore, it is important to develop oral mucosa vaccines that can elicit effective mucosal 
immune responses against PEDV infection. Nevertheless, to reach the mucosal immunity-related sites in sufficient amounts, oral mucosal vaccines need to withstand harsh digestive environments, which are proving to be a big challenge for mucosal vaccine development [3]; therefore, effective antigen delivery vehicles and the adjuvant limit the availability of oral mucosal vaccines for stimulation of immunity [4].

Currently, there is increasing interest in the development of lactic acid bacteria (LAB)-based oral vaccines against enteric viruses; this approach is crucial for the effective induction of a mucosal immune response. Lactobacillus strains have many characteristics that make them promising candidates as delivery systems for presenting compounds with antigens of interest to the mucosa, in particular vaccines [5]; for example, Lactobacilli can survive in (and colonize) the upper gastrointestinal tract and exert an intrinsic adjuvant activity [6,7]. Moreover, recent reports have shown that Lactobacillus species can effectively elicit production of secretory immunoglobulin A (SIgA), induce anti-inflammatory responses, activate innate cells, and regulate the balance between $\mathrm{T}$ cell subset responses [8,9]. On the other hand, in order to enhance the deliver efficiency of vaccine antigens to mucosal immune tissues via oral administration, a dendritic cell (DC)-targeting mucosal vaccine was suggested as a realistic approach for oral vaccination to induce high mucosal immune responses against infection [10]. DCs, widely distributed beneath the gastrointestinal epithelium, are professional antigen-presenting cells serving as a portal for virus invasion [11]; therefore, DCs are an early target for virus attachment. Intestinal DC subsets regulate of the intestinalimmune homeostasis through linking humoral and cellular immune responses [12]. It was confirmed that an intestinal DC-targeting oral vaccine could elicit highly efficient antigen-specific immune responses, protecting the mucosal membrane against pathogen infection [13]. Currently, the vaccine strategy of using Lactobacillus to express DC-targeting peptide (DCpep) conjugated with Bacillus anthracis PA antigen [14] and Newcastle disease virus HN antigen has been investigated to show improved immunogenicity.

Moreover, secretory $\operatorname{Ig} \mathrm{A}(\mathrm{SIg}$ ) is the predominant antibody isotype on all mucosal surfaces and prevents bacteria and viruses from infecting the intestinal mucosal barrier [15]. SIgA establishes the first line of defense on the mucosal surface to block adhesion and invasion of infectious agents [16]. Because the mucosal surface is the initial infection site for PEDV, especially in the intestinal mucosa, it would be interesting to develop oral mucosal vaccines that elicit an effective mucosal immune response against PEDV infection. The Spike (S) glycoprotein of PEDV that mediates receptor binding and membrane fusion [17] harbors several neutralizing epitopes [18], particularly the core neutralizing epitope (COE), which can induce neutralizing antibodies against PEDV [19]. The COE has been successfully expressed for vaccine purposes in plants [20] and in lactic acid bacteria [21].

In the present study, a recombinant Lactobacillus casei expressing the DC-targeting peptide conjugated with PEDV COE antigen was developed, and its immunogenicity upon administration as an oral vaccine was evaluated.

\section{Materials and Methods}

\subsection{Bacterial Strain, Virus, and Plasmid}

Lactobacillus casei ATCC 393 (L. casei 393) was cultured in our laboratory in de Man, Rogosa and Sharp (MRS) broth at $37^{\circ} \mathrm{C}$ without shaking. PEDV LJB/15 strain was isolated and identified from clinical samples by our laboratory and was propagated in Vero cells at $37{ }^{\circ} \mathrm{C}$ with $5 \% \mathrm{CO}_{2}$. The constitutive expression plasmid pPG-T7g10-PPT (Figure 1Aa), was constructed in our laboratory. 


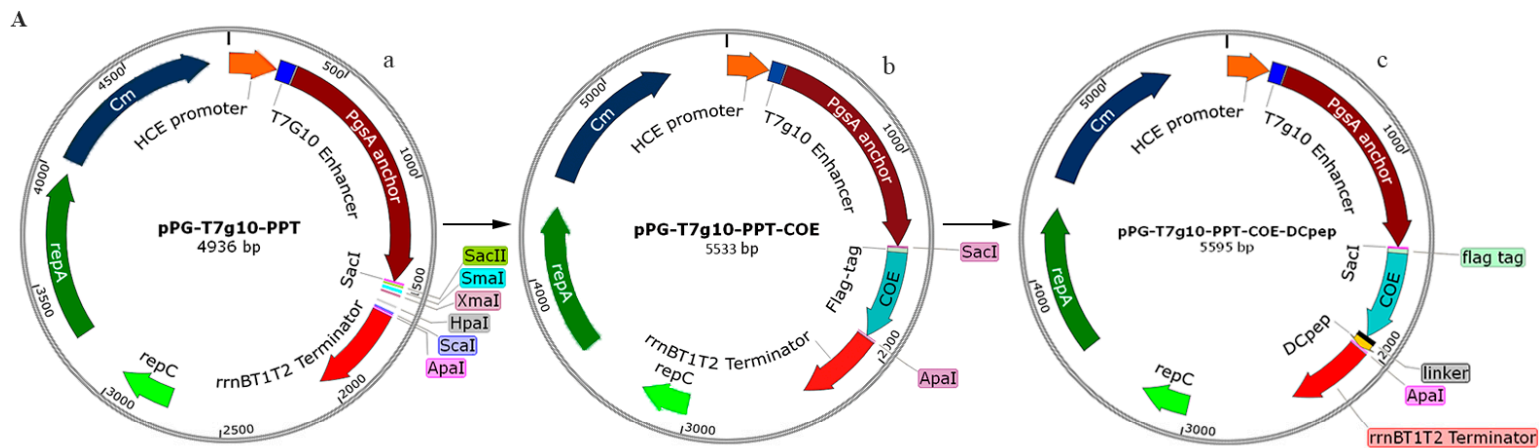

B

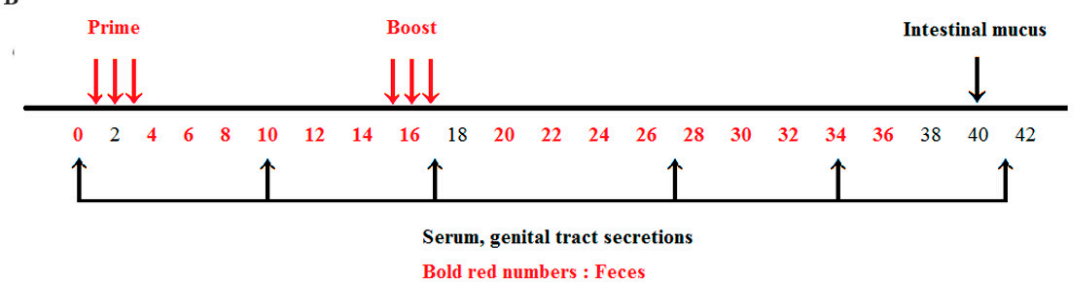

Figure 1. (A) Schematic diagram of the construction of recombinant plasmids. (a) The constitutive cell surface expression plasmid pPG-T7g10-PPT, (b) recombinant plasmid pPG-T7g10-PPT-COE containing core neutralizing epitope (COE), (c) recombinant plasmid pPG-T7g10-PPT-COE-DCpep containing the fusion gene COE-DC-targeting peptide (DCpep); (B) Details of the immunization schedule for recombinant lactic acid bacteria and sampling post-immunization. Red arrows indicate the time points of primary immunization and booster immunization; black arrow (above the line) indicate the time point of intestinal mucus collection, and black arrows (below the line) indicate the time points of serum and genital tract secretions collection.

\subsection{Construction of Recombinant Lactic Acid Bacteria}

A schematic diagram for the construction of recombinant plasmids is shown in Figure 1A. In brief, following genomic RNA extraction from PEDV propagated in Vero cells, the gene encoding the COE antigen of PEDV was obtained by reverse transcription (RT)-PCR, and the gene encoding DCpep was then fused to $3^{\prime}$ terminus of the COE gene by fusion PCR. DC-targeting peptides (DCpep) that specifically bound to human DCs after screening a 12-mer peptide phage display library [7]. In this study, primer pairs F1/P1 and F1/DCpep (Table 1) were used to amplify COE gene and COE-DCpep, respectively.The gene $C O E$ and the fusion gene $C O E-D C p e p$ were then cloned into the expression plasmid pPG-T7g10-PPT, giving rise to recombinant plasmids pPG-T7g10-PPT-COE (Figure 1Ab) and pPG-T7g10-PPT-COE-DCpep (Figure 1Ac), respectively. Details of the primers used in this study are listed in Table 1. The recombinant plasmids pPG-T7g10-PPT-COE and pPG-T7g10-PPT-COE-DCpep were then transformed into L. casei 393 competent cells by electroporation [22], to generate recombinant strains pPG-COE/L393 and pPG-COE-DCpep/L393, respectively. 
Table 1. Details of primers used in this study.

\begin{tabular}{|c|c|c|c|c|}
\hline Target & ID & Primer Sequence $\left(5^{\prime}-3^{\prime}\right)$ & PCR Size & $\begin{array}{c}\text { Accession } \\
\text { No./Reference }\end{array}$ \\
\hline \multirow[t]{2}{*}{$\mathrm{COE}$} & F1 & $\begin{array}{l}\text { GAGCTC }{ }^{\mathrm{a}} \text { GATTATAAGGATGACGATGACA } \\
\text { AG }^{\mathrm{b}} \text { AAGCTTGTTACTTTGCCATCGTTT }\end{array}$ & \multirow{2}{*}{$465 \mathrm{bp}$} & \multirow[t]{2}{*}{ JX512907 } \\
\hline & P1 & GGGCCC ${ }^{a}$ TCAAACGTCCGTGACACCTTC & & \\
\hline \multirow[t]{2}{*}{ COE-DCpep } & $\mathrm{F} 1$ & $\begin{array}{l}\text { GAGCTC }{ }^{\mathrm{a}} \text { GATTATAAGGATGACGATGACA } \\
\text { AG }^{\mathrm{b}} \text { AAGCTTGTTACTTTGCCATCGTTT }\end{array}$ & \multirow{2}{*}{$507 \mathrm{bp}$} & Shi et al., 2016 [23] \\
\hline & DCpep & $\begin{array}{c}\text { GGGCCC }^{\mathrm{a}} \text { TTATGGACGTTGTGGTGTTGAATGA } \\
\text { TATGATGGATAAAA' }\end{array}$ & & \\
\hline
\end{tabular}

${ }^{a}$ Restriction enzyme recognition sites used for cloning; ${ }^{b}$ Flag tag are shown in bold; ${ }^{\mathrm{c}}$ DCpep sequences are shown in italic.

\subsection{Protein Expression}

The bacterial strains pPG-COE-DCpep/L393, pPG-COE/L393, pPG/L393 and L. casei 393 were cultured overnight in MRS broth and harvested by centrifugation at $9000 \times g$ for $10 \mathrm{~min}$ at $4{ }^{\circ} \mathrm{C}$. After cell lysis and centrifugation, the supernatant was subjected to $12 \%$ sodium dodecyl sulfate-polyacrylamide gel electrophoresis (SDS-PAGE) and western blot assay. The proteins in the supernatant were separated by SDS-PAGE, electrotransferred onto PVDF membranes (Millipore, Milford, MA, USA), and the immunoblot was then developed using a mouse anti-COE monoclonal antibody (dilution at 1:500) prepared in our laboratory as the primary antibody, and horseradish peroxidase (HRP)-conjugated goat anti-mouse IgG antibody (dilution at 1:3000) (Sigma, Ronkonkoma, NY, USA) as the secondary antibody. The results were then visualized using a chemiluminescent substrate reagent (Thermo Scientific, Durham, NC, USA) according to the manufacturer's instructions.

Moreover, in order to further analyze whether the protein of interest was displayed on the cell surface of the recombinant strains pPG-COE-DCpep/L393 and pPG-COE/L393, an immunofluorescence assay was performed according to a previously described method [24]. Briefly, the recombinant strains pPG-COE-DCpep/L393 and pPG-COE/L393 were cultured overnight in MRS at $37^{\circ} \mathrm{C}$. After centrifugation at $12,000 \times \mathrm{g}$ for $5 \mathrm{~min}$, the cell pellets were incubated with mouse anti-COE monoclonal antibody (dilution at 1:100) and fluorescein isothiocyanate (FITC)-labeled goat anti-mouse IgG (Invitrogen, Carlsbad, CA, USA) (diluted at 1:4000). After washing three times with phosphate buffered saline (PBS), the nuclei were counterstained with $4^{\prime}, 6^{\prime}$-diamidino-2-phenylindole (DAPI) (Invitrogen, Carlsbad, CA, USA) for $30 \mathrm{~min}$ at $4{ }^{\circ} \mathrm{C}$. The pellets were washed thrice, resuspended in $1 \mathrm{~mL}$ PBS and smeared on a microscope slide. Fluorescence images were acquired by laser confocal microscopy (Zeiss, Oberkochen, Germany).

\subsection{Immunization and Specimen Collection}

All the experimental procedures and animal management procedures were approved by the Institutional Committee of the Northeast Agricultural University for the Animal Experiments (2016NEFU-315, 13 April 2017), Harbin, China. Six-week-old female specific pathogen-free (SPF) BALB/c mice $(n=60)$ were obtained from Liaoning Changsheng Biotechnology Co., Ltd. (Benxi, China) and kept under SPF conditions for one week with free access to a standard chow diet and water in accordance with institutional guidelines.Prior to oral administration, the recombinant strains weregrown in MRS broth at $37{ }^{\circ} \mathrm{C}$ for $12 \mathrm{~h}$, and the cells were then washed twice with PBS, and resuspended in PBS to a final concentration of $10^{10} \mathrm{CFU} / \mathrm{mL}$. The mice were orally dosed with $100 \mu \mathrm{L}$ of pPG-COE-DCpep/L393 and pPG-COE/L393groups (15 mice per group). The control groups of mice (15 mice per group) were orally administered with an equivalent dose of pPG/L393 and PBS. The immunization protocol was performed on three consecutive days (days 1,2, and 3), and a booster immunization was given on days 15,16, and 17 (Figure 1B). Specimen collection was then performed at different time points post immunization (Figure 1B). Serum samples were collected from immunized 
mice on days $0,10,17,27,34$, and 41 after immunization and stored at $-20^{\circ} \mathrm{C}$ until used. Genital tract secretions were collected on days $0,10,17,27,34$, and 41 after primary immunization and stored at $-40^{\circ} \mathrm{C}$ until analyzed by ELISA for SIgA levels. Fecal samples were used for detecting SIgA antibody were collected and treated according to methods described previously with slight modifications [25]. In brief, $0.1 \mathrm{~g}$ of fecal pellets were sampled and subsequently suspended in $400 \mu \mathrm{L}$ of PBS containing $1 \mathrm{mmol} / \mathrm{L}$ phenylmethylsulfonyl fluoride (Sigma, Ronkonkoma, NY, USA) and 1\% bovine serum albumin (BSA). Then, after incubating at $4{ }^{\circ} \mathrm{C}$ for $16 \mathrm{~h}$ and centrifugation at $12,000 \times g$ for $5 \mathrm{~min}$, the supernatants were stored at $-20^{\circ} \mathrm{C}$ until used. In addition, on days 0 and 40 , the intestinal mucus samples was gently scraped from the excised intestinal tissue with HEPES buffer, and clarified by centrifugation as described previously [26].

\subsection{Activation of DC Costimulatory Molecules}

To evaluate the potential effect of pPG-COE-DCpep/L393 on DCs, murine mesenteric lymph nodes (MLN) cells were collected at $36 \mathrm{~h}$ after orally inoculated with pPG-COE/L393, pPG-COE-DCpep/L393 according to methods described previously [27]. Briefly, MLN cells were prepared using a $70 \mathrm{mM}$ pore filter (BD Falcon, Franklin Lakes, NJ, USA) by gentle crushing of organs. Single MLN cell suspensions were incubated with CD16/CD32 (BD PharMingen, San Diego, CA, USA) to block $F_{c}$ receptors ( $\left.F C R\right)$. After cell suspensions were washed and resuspended in complete RPMI 1640, the cells were analyzed for DC activation by staining with fluorescein isothiocyanate (FITC)-conjugated surface molecule CD11c, CD80, CD86 and MHC II (BD Biosciences, San Diego, CA, USA). Samples were examined with a FACSCalibur cytometer.

\subsection{ELISA}

The levels of anti-PEDV specific IgG antibody in the sera and SIgA antibody in the feces, genital tract secretions, and intestinal mucus samples collected from immunized mice at the indicated time points were determined by ELISA. Briefly, a polystyrene microtiter plate was coated overnight at $4{ }^{\circ} \mathrm{C}$ with PEDV propagated in Vero cells. After washing thrice with PBS-0.1\% Tween 20, the plate was blocked with $5 \%$ skimmed milk at $37^{\circ} \mathrm{C}$ for $2 \mathrm{~h}$. After washing, the collected immune samples were added into the plate in triplicate and incubated at $37^{\circ} \mathrm{C}$ for $1 \mathrm{~h}$. After washing, HRP-conjugated goat anti-mouse IgG/IgA-specific antibody (Invitrogen, Carlsbad, CA, USA) was added to the plate and incubated at $37{ }^{\circ} \mathrm{C}$ for $1 \mathrm{~h}$. Furthermore, the expression of DCpep was verified by ELISA with COE-DCpep antiserum, obtained from mice immunized with pPG-COE-DCpep/L393. Briefly, a polystyrene microtiter plate was coated with synthetic DCpep protein. After being washed and blocked, the two-fold serially dilutions of COE-DCpep antiserum were added into the plate in triplicate, and then HRP-conjugated goat anti-mouse IgG-specific antibody (Invitrogen, Carlsbad, CA, USA) was added to the plate.The substrate o-phenylenediaminedihydrochloride (Sigma, Ronkonkoma, NY, USA) was used for color development followed by determination of absorbance at $490 \mathrm{~nm}$.

\subsection{Determination of PEDV Neutralizing Activity of Antibodies}

For determining the PEDV neutralizing activity of the serum antibodies obtained from the immunized mice, $50 \mu \mathrm{L}$ of sera samples serially diluted two-fold, were each mixed with $50 \mu \mathrm{L}$ of 200 $50 \%$ tissue culture infective dose (TCID $\left.{ }_{50}\right)$ PEDV propagated in Vero cells and incubated at $37^{\circ} \mathrm{C}$ for $1 \mathrm{~h}$; the mixture was then transferred to Vero cell monolayers in 96-wellplates. After incubation at $37^{\circ} \mathrm{C}$ in a $5 \% \mathrm{CO}_{2}$ atmosphere for 3 days, PEDV-specific cytopathic effects (CPE) were observed. In this work, three technical replicates and eight biological replicates per sample were performed, and the positive serum control, negative serum control, virus control, and blank control were designed in parallel. 


\subsection{Lymphocyte Proliferation and Cytokine Detection}

On day 40 after primary immunization, five mice from each group were sacrificed for preparing splenocytes under aseptic conditions to perform the lymphocyte proliferation assay, as previously described [28]. In brief, the splenocytes in quadruplicates at a concentration of $5 \times 10^{6}$ cells $/ \mathrm{mL}$ were cultured in RPMI- 1640 plus $20 \%$ fetal calf serum at $37{ }^{\circ} \mathrm{C}$ with $5 \% \mathrm{CO}_{2}$ in a 96 -well plate. The cells were stimulated with $0.5 \mu \mathrm{g} / \mathrm{mL}$ and $5 \mu \mathrm{g} / \mathrm{mL}$ of purified PEDV COE protein (specific antigen stimulation) for $60 \mathrm{~h}$ at $37^{\circ} \mathrm{C}$. In parallel, stimulation with $5 \mu \mathrm{g} / \mathrm{mL}$ of concanavalin A (ConA) and culture medium was used as the positive control and negative control, respectively. With absorbance measured at $490 \mathrm{~nm}$, lymphocyte proliferation was evaluated using the CellTiter 96 AQueous Non-Radioactive Cell Proliferation Assay (Promega, Madison, WI, USA)according to the manufacturer's instruction.The stimulation index was calculated as follows: the mean reading of triplicate antigen stimulation wells divided by the mean reading of triplicate negative control wells.

For detecting the interferon-gamma (IFN- $\gamma$ ) and interleukin-4 (IL-4) levels in the culture supernatant of splenocytes, the culture supernatants were collected after antigen stimulation for $60 \mathrm{~h}$, and used in OptEIA Set Mouse IFN- $\gamma$ and IL-4 (Biosource InternationalInc., Camarillo, CA, USA) antigen-capture ELISA according to the manufacturer's instructions. All assays were performed in quadruplicate. In the culture supernatants, the concentration of IFN- $\gamma$ and IL-4was calculated using a linear-regression equation and the absorbance values of the standards [29].

\subsection{Statistical Analysis}

Data are shown as the means \pm standard errors of three replicates per test in a single experiment repeated thrice. GraphPad Prism V5.0 (San Diego, CA, USA) was used to perform statistical analyses. Tukey's multiple comparison tests and one-way analysis of variance (ANOVA) were used to analyze the significance of the difference between means. $p$-Values less than $0.05(p<0.05)$ were considered significant and $p$-values less than $0.01(p<0.01)$ as highly significant.

\section{Results}

\subsection{Identification of the Protein of Interest Expressed by Recombinant L. casei}

Overnight cultures of pPG-COE-DCpep/L393, pPG-COE/L393, pPG/L393, and L. casei 393 were centrifuged, lysed, and analyzed by western blot assay. As shown in Figure 2A, a specific immunoreactive band of the expected size was observed in the lysate of the recombinant strain pPG-COE/L393 (lane 1) and pPG-COE-DCpep/L393 (lane 3), respectively, but not in pPG/L393 (lane 2) and L. casei 393 (lane 4), indicating that the protein of interest was efficiently expressed by the genetically engineered L. casei 393 strains. Moreover, an immunofluorescence assay was used to analyze the cell surface display of the protein of interest. Cells of overnight cultured L. casei 393, pPG/L393, pPG-COE/L393, and pPG-COE-DCpep/L393 were incubated with mouse anti-COE monoclonal antibody and FITC-conjugated goat anti-mouse IgG antibody, and then observed by laser confocal microscopy. As shown in Figure 3, green fluorescence observed on the cell surface of pPG-COE/L393 and pPG-COE-DCpep/L393, but not on pPG/L393 and L. casei 393. The results indicated that the protein of interest was expressed and displayed on the cell surface of pPG-COE/L393 andpPG-COE-DCpep/L393.

As shown in Figure 2B, DCpep protein can be detected with COE-DCpep antiserum which wasdiluted at 1:2-1:64. With the increase of dilution of COE-DCpep antiserum, the immunoreactive is weakened. Moreover, we showed that there is no cross-reactivity between the mouse anti-COE monoclonal antibody and synthetic DCpep protein. 
A

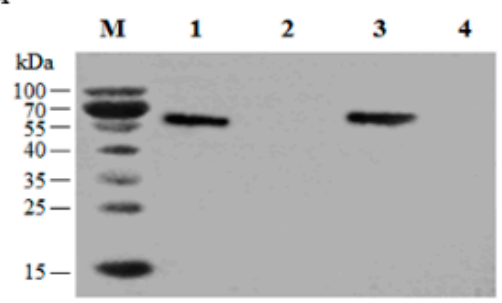

C

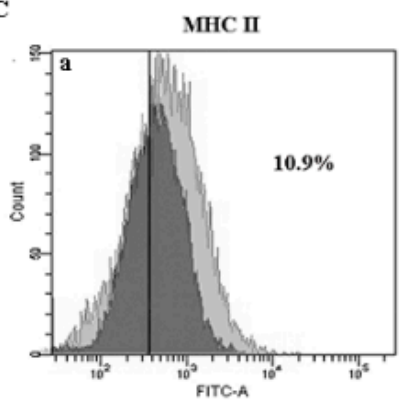

B

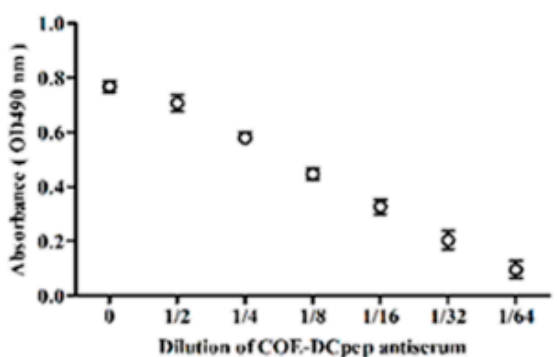

Figure 2. Expression of the protein of interest identified by western blot and ELISA $(\mathbf{A}, \mathbf{B})$, and activation of DC costimulatory molecules by L. casei 393 expressing pPG-COE-DCpep (C). The proteins in the lysate of overnight cultured L. casei 393, pPG/L393, pPG-COE/L393, and pPG-COE-DCpep/L393 were separated by sodium dodecyl sulfate-polyacrylamide gel electrophoresis (SDS-PAGE) followed by identification by western blot. Immunoblot with the expected sizes were respectively observed for recombinant strain pPG-COE/L393 (lane 1) and pPG-COE-DCpep/L393 (lane 3), but not for pPG/L393 (lane 2) and L. casei 393 (lane 4) (Figure 2A). M: protein molecular weight maker. Expression of DCpep protein were analyzed by ELISA (Figure $2 \mathrm{~B}$ ) in miceimmunized the recombinant $L$. casei, using synthetic DCpep protein as the coating antigen. DCs were isolated from the mesenteric lymph nodes (MLNs) of mice immunized with pPG-COE/L393, pPG-COE-DCpep/L393 at $36 \mathrm{~h}$, and the expression levels of histocompatibility complex (MHC) II (Figure 2Ca), CD86 (Figure 2Cb) and CD80 (Figure 2Cc) were determinedby flow cytometry. 


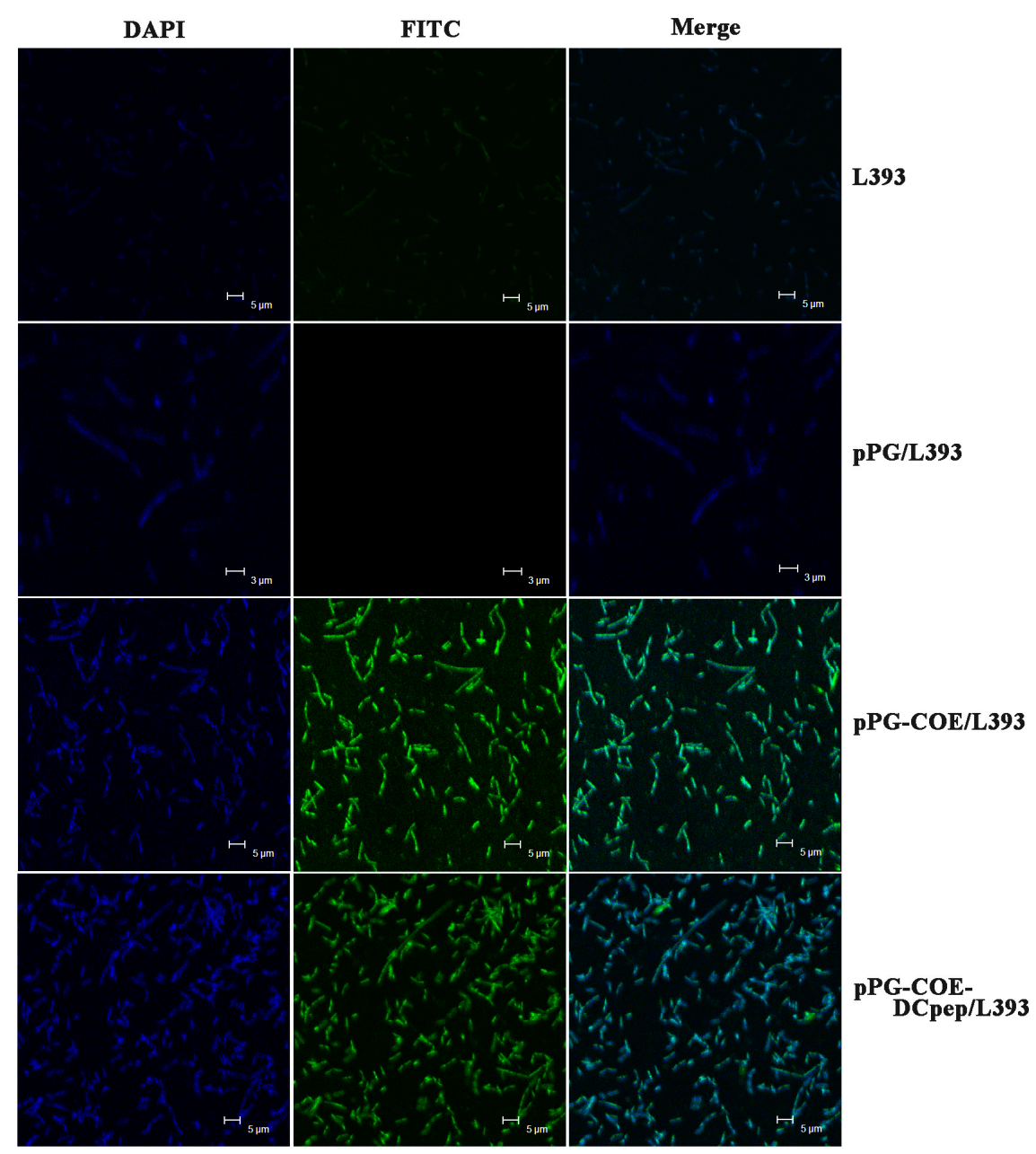

Figure 3. Cell surface display of protein of interest analyzed by immunofluorescence assay. Green fluorescence was only observed on the cell surface of pPG-COE/L393 and pPG-COE-DCpep/L393, and not on pPG/L393 and L. casei 393, indicating that the protein of interest was expressed and displayed on the cell surface of pPG-COE/L393 and pPG-COE-DCpep/L393.

\subsection{Activation of DC Costimulatory Molecules by pPG-COE-DCpep/L393}

To evaluate the potential effect of pPG-COE-DCpep/L393 on DCs, mice were orally immunized with pPG-COE/L393 and pPG-COE-DCpep/L393. The costimulatory molecules of DC were analyzed by staining with fluorescein isothiocyanate (FITC)-conjugated CD80, CD86 and histocompatibility complex (MHC) II, as shown in Figure 2C. The results showed that compared to the pPG-COE/L393, pPG-COE-DCpep/L393 induced a significantly increased in the surface expression of CD80 ${ }^{+}, \mathrm{CD}^{+} 6^{+}$ and $\mathrm{MHCII}^{+}$on $\mathrm{CD} 11 \mathrm{c}^{+}$cells at $36 \mathrm{~h}$ post-vaccination in mice (the ratio (\%) of the DC marker CD11 $\mathrm{c}^{+}$ was $>80 \%$ ) which indicated that the oral vaccination of mice with pPG-COE-DCpep/L393 effectively elicited the activation of DCs in the MLNs.

\subsection{IgG Levels Induced by Recombinant L. casei 393 via Oral Immunization}

The level of anti-PEDV IgG antibodies in mice induced by pPG-COE/L393 and pPG-COE-DCpep/L393 was determined by ELISA. As shown in Figure 4A, from the tenth day after primary vaccination, a significant level of anti-PEDV specific IgG antibody was induced in mice that were orally administered with pPG-COE/L393 and pPG-COE-DCpep/L393 $(p<0.01)$ compared to the pPG/L393 and PBS groups. Moreover, after the booster immunization, a higher level of anti-PEDV IgG antibody was elicited in mice orally immunized with pPG-COE-DCpep/L393 
$(p<0.01)$ compared to the pPG-COE/L393 group, which indicated that the DC-targeting peptide used as a vaccine adjuvant could enhance the immunogenicity of the antigen and promote the vaccine immune efficiency. However, there was no significant difference in the IgG antibody level of the control groups before and after immunization.

Moreover, the anti-PEDV neutralizing activity of the serum antibody obtained from immunized mice was determined (Figure 4B). Results showed that the antibody response in mice that received pPG-COE-DCpep/L393 possessed a stronger anti-PEDV neutralizing activity (1:24) than that in mice orally administered with pPG-COE/L393 (1:6), pPG/L393 (1:2) and PBS (1:2). As might be expected, the L. casei oral vaccine (pPG-COE-DCpep/L393) expressing the DC-targeting peptide fused with PEDV COE antigen could effectively elicit an anti-PEDV immune response in vivo.

A

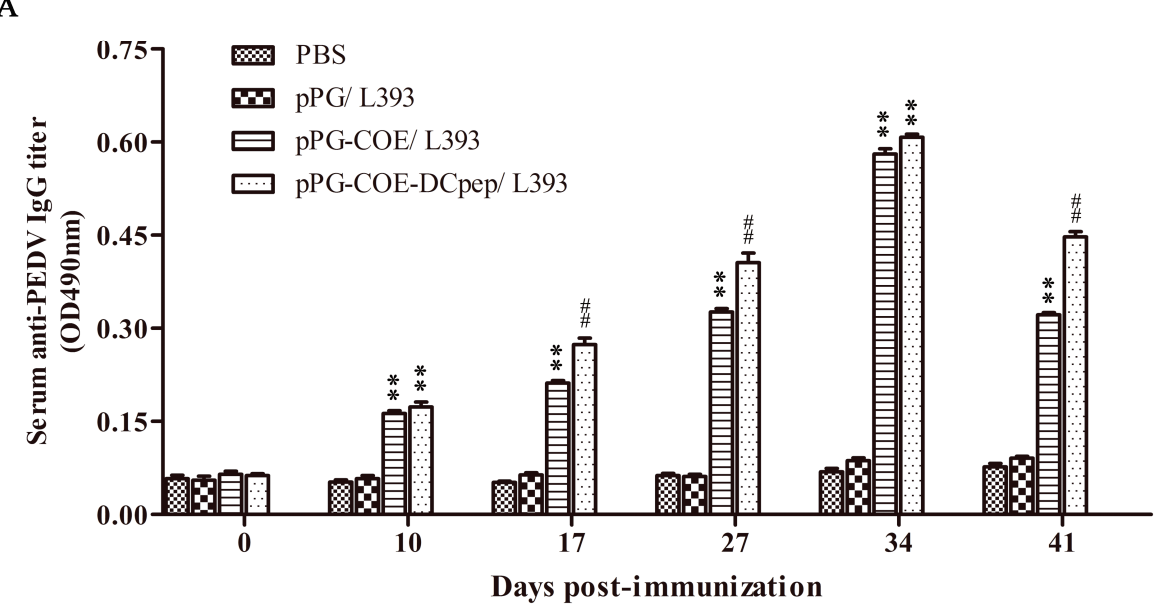

B

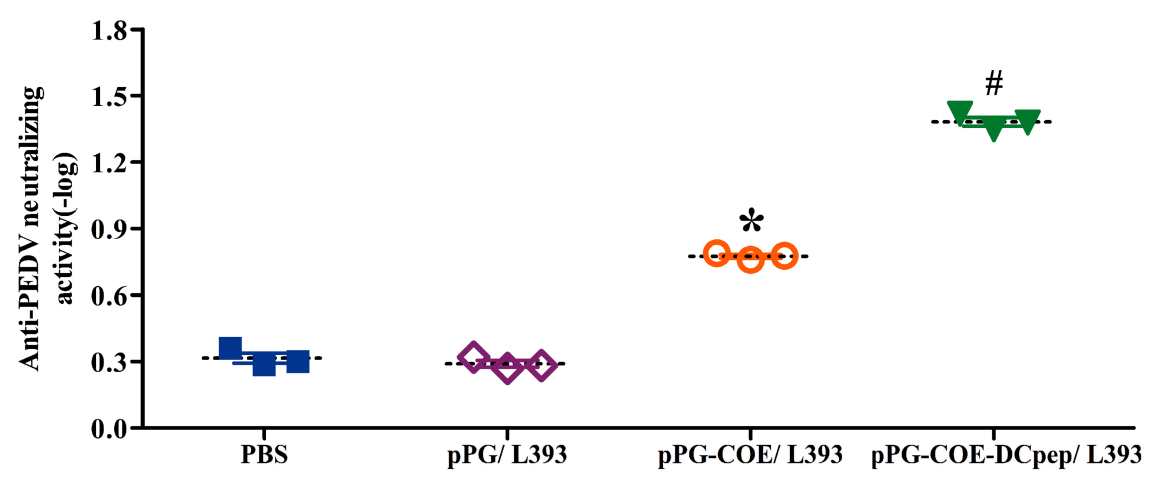

Figure 4. Determination of anti-porcine epidemic diarrhea virus (PEDV) specific IgG antibody and anti-PEDV neutralizing activity in mice post-immunization. Measurement of anti-PEDV IgG antibody (A) and anti-PEDV neutralizing activity (B) in sera from immunized mice by ELISA using PEDV as the coating antigen. Bars represent the mean \pm standard error value of each group $\left({ }^{*} p<0.05\right.$, ${ }_{*}^{*} p<0.01$ compared to the control groups: pPG/L393 and PBS; ${ }^{\#} p<0.05,{ }_{*}^{*} p<0.01$ compared to group: pPG-COE/L393).

\subsection{SIgA Levels Induced by Recombinant L. casei 393 via Oral Immunization}

The intestinal mucosa cell-mediated immune response was further evaluated by measuring the specific anti-PEDV SIgA antibody in the feces, genital tract, and intestinal mucus collected from immunized mice post-immunization via ELISA. The mucosal SIgA levels increased after oral immunization with pPG-COE/L393 and pPG-COE-DCpep/L393 in the feces (Figure 5A), genital tract (Figure 5B), and the intestinal mucus (Figure 5C) $(p<0.01)$ compared to those in the control groups of mice orally immunized with pPG/L393 and PBS. Anti-PEDV SIgA in the feces could be detected 
as early as the sixth day post-immunization with pPG-COE-DCpep/L393 and the eighth day after primary vaccination with PPG-COE/L393, respectively; following a booster immunization, the SIgA levels significantly increased. There was no significant difference in the SIgA level observed in the control groups of mice before and after immunization $(p>0.05)$. In contrast, the level of SIgA antibody induced by pPG-COE-DCpep/L393 via oral immunization was significantly higher than that induced by pPG-COE/L393.

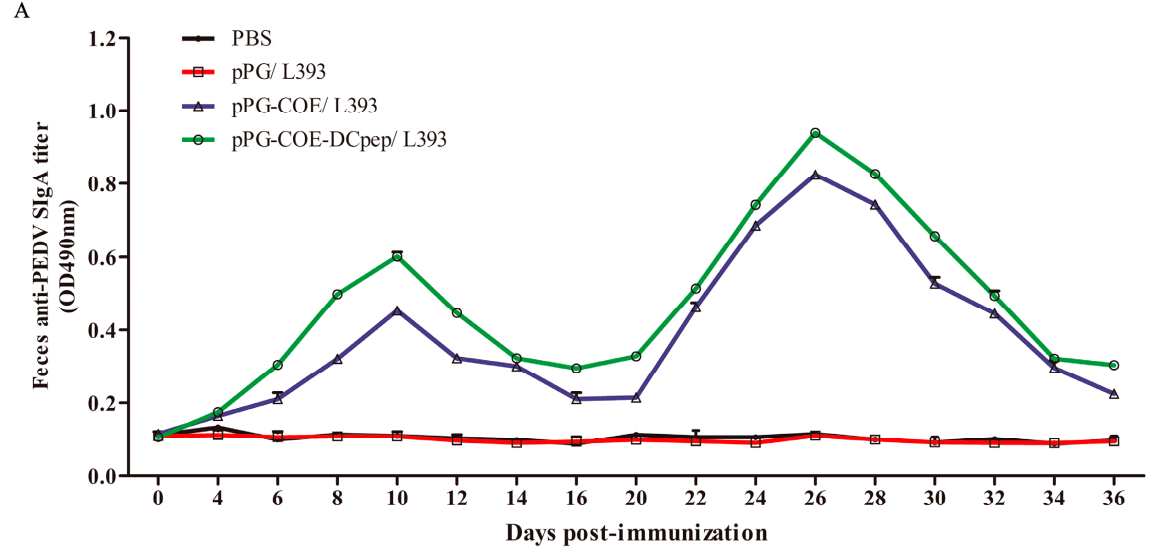

B

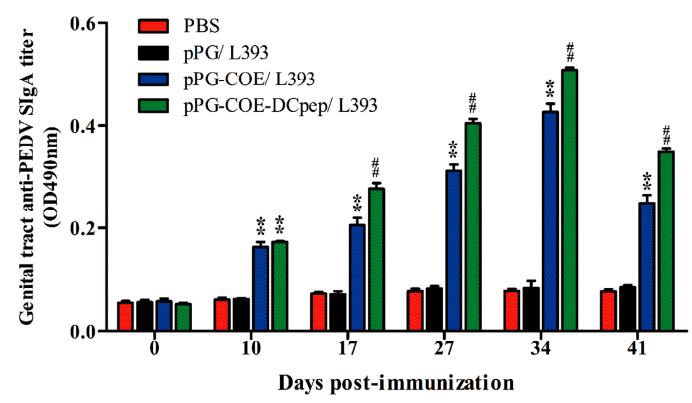

$\mathrm{C}$

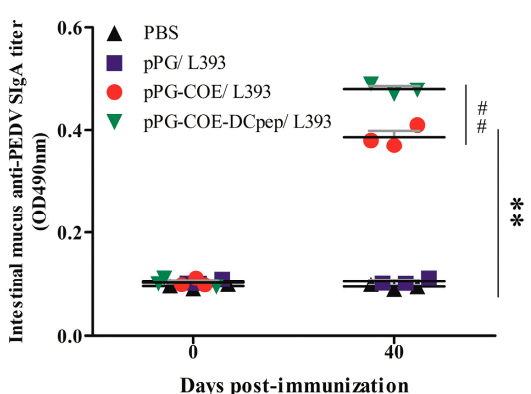

Figure 5. Determination of anti-PEDV mucosal SIgA antibody in feces (A), genital tract (B) and intestinal mucus $(\mathbf{C})$ by ELISA using PEDV as the coating antigen. Bars represent the mean \pm standard error value of each group $\left({ }^{*} p<0.05,{ }_{*}^{*} p<0.01\right.$ compared to the control groups pPG/L393 and PBS; ${ }^{\#} p$ $<0.05,{ }^{\#} p<0.01$ compared to group pPG-COE/L393 in Figure 5B,C).

\subsection{Lymphocyte Proliferation}

Splenocytes were isolated from immunized mice and restimulated with the PEDV COE protein in vitro to test the lymphocyte proliferation response by 3-(4,5-dimethylthiazol-2-yl)-2,5diphenyltetrazolium bromide (MTT) assay using Con A as the positive control and cell culture medium as negative control. As shown in Figure 6, upon stimulation with the purified PEDV COE protein, the stimulation index markedly increased in the group immunized with pPG-COE-DCpep/L393 compared to that in the groups immunized with pPG-COE/L393 $(p<0.05)$, pPG/L393 $(p<0.01)$ and PBS $(p<0.01)$, and showed a dose-dependent response. 


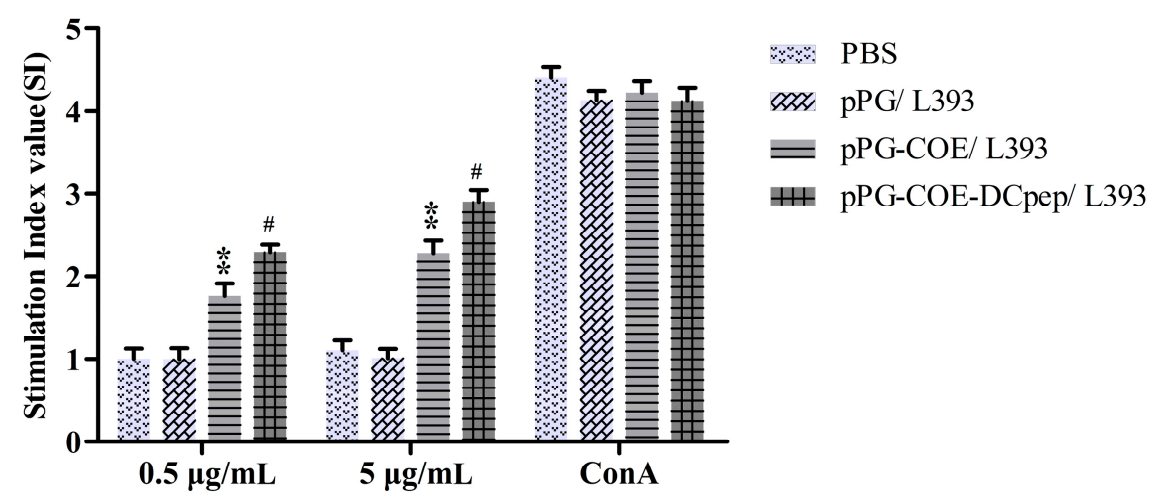

Figure 6. Lymphocyte proliferation in immunized mice determined by 3-(4,5-dimethylthiazol-2-yl)2,5-diphenyltetrazolium bromide (MTT) assay in response to PEDV COE protein as a stimulating agent. Bars represent the mean \pm standard error value of each group $\left({ }^{*} p<0.05,{ }_{*}^{*} p<0.01\right.$ compared to the control groups: pPG/L393 and PBS; ${ }^{\#} p<0.05,{ }_{\#}^{\#} p<0.01$ compared to group: pPG-COE/L393).

\subsection{Cytokine Levels}

As shown in Figure 7, in response to the PEDV COE antigen, splenocytes obtained from mice orally administered with pPG-COE-DCpep/L393 produced significant higher levels of the Th1-associated cytokine IFN- $\gamma$ and the Th2-associated cytokine IL-4 than those in mice receiving pPG-COE/L393 $(p<0.01)$.

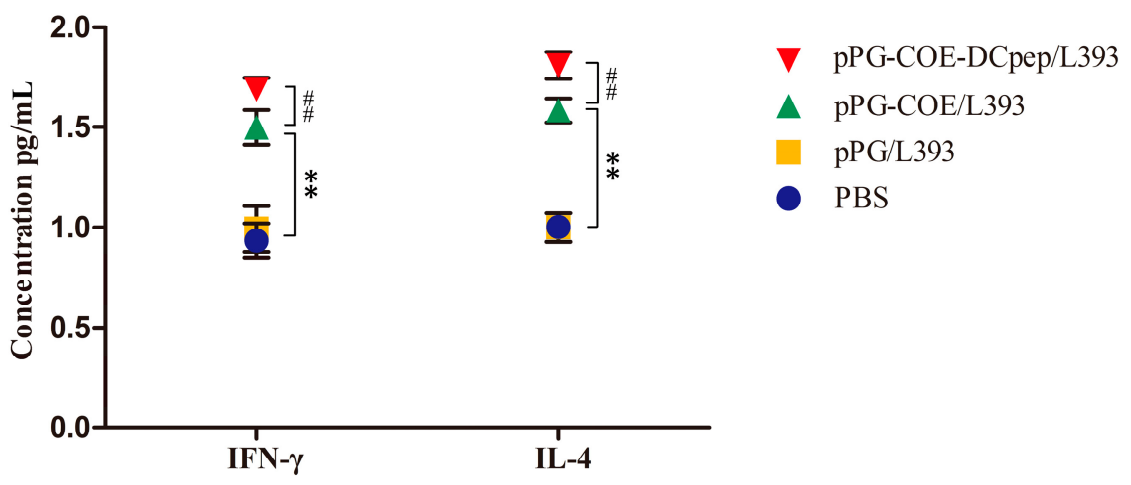

Figure 7. Determination of cytokines produced by splenocytes of mice orally administered with pPG-COE-DCpep/L393, pPG-COE/L393, pPG/L393, and PBS, respectively. Bars represent the mean \pm standard error value of each group $\left({ }^{*} p<0.05,{ }_{*}^{*} p<0.01\right.$ compared to the control groups pPG/L393 and PBS; ${ }^{\#} p<0.05,{ }_{\text {\# }} p<0.01$ compared to group $\left.\mathrm{pPG}-\mathrm{COE} / \mathrm{L} 393\right)$.

\section{Discussion}

Currently, porcine epidemic diarrhea caused by PEDV has become the one of the important swine diseases, resulting in huge economic loses for the pig industry. Since 2013, porcine epidemic diarrhea has spread throughout the United States and has greatly damaged the American pork industry [30]. The vaccination failure and high death rate seen in the 2010 Chinese outbreak with the re-emergence of a more highly virulent PEDV may have been due to changes in the antigenicity of PEDV based on amino acid mutations [31]. The emergence and re-emergence of PEDV suggests that it is able to elude current vaccine strategies [32]. However, the neutralizing antigenic epitopes of S protein of PEDV have been showed high cross-protection against variant strains [33]. Therefore, the use of core neutralizing epitope (COE) of PEDV isolates $S$ protein to develop PED vaccines should be a useful approach for control of PED epidemics in the region. In this study, a PEDV isolate LJB/15 was used 
and the nucleotide sequence encoding the COE of PEDV LJB/15 showed more than $98 \%$ identity with other PEDV strains isolated in China in recent years.

The mucosal immune system, based mostly on SIgA, is the first and a major barrier against invading pathogens targeting the gastrointestinal tract [23]. Therefore, it is necessary to develop oral mucosal vaccines that can induce strong and protective immune responses in the mucosa (to prevent initial infection and pathogen replication), predominantly by means of SigA [34]. L. casei 393, as the antigen delivery carrier used in our study, can adhere to and colonize intestinal mucosa of murine and pigs and to tolerate bile, thus serving as a promising candidate for the delivery of antigenic material to the mucosa in some vaccines $[35,36]$. In order to enhance the delivery efficiency of the antigen of interest and reduce the number of vaccinations and the quantity of lactic acid bacteria required for oral administration, intestinal DC-targeting mucosal vaccines were suggested as a promising approach of oral vaccination to induce high mucosal immune responses [10].

In this study, a genetically engineered Lactobacillus casei oral vaccine (pPG-COE-DCpep/L393) expressing a DC-targeting peptide fused with PEDV COE antigen was developed. Expression of the fusion protein was identified by western blot and immunofluorescence assays. The immunogenicity of the orally administered pPG-COE-DCpep/L393 was evaluated in mice, and the results showed that oral immunization with pPG-COE-DCpep/L393 can induce a significant level of anti-PEDV systemic IgG and mucosal SIgA antibody responses, indicating a potential vaccine strategy against PEDV infection.

The antigens of interest intracellularly biosynthesized by lactic acid bacteria could not effectively induce antigen-specific immune responses via oral administration, due mainly to the target antigens cannot effectively interact with intestinal mucosa tissues, in particular DC cells [37]. Thus, in this study, we used the pPG-T7g10-PPT expression vector that was a constitutive cell surface expression plasmid developed by our lab to construct recombinant lactic acid bacteria for the delivery of vaccine antigen. Remarkably, pPG-T7g10-PPT exhibiting a significant advantage as compared with inducible gene expression systems, which require the use of an inductive agent. Additionally, we used phosphatidylglycerol phosphate synthase (pgsA) from Bacillus subtilis subsp. chungkookjang as an anchor protein and fused the COE protein to its $C$ terminus within pPG-T7g10-PPT expression vector so that it can anchor the protein to the bacterial surface without a sortase [38], thereby enhancing the immunogenicity. Therefore, in this study, the constitutive cell surface expression system serves as a powerful tool for the construction of recombinant Lactobacillus oral live vaccines.

It is now clear that the intestinal mucosa is the primary site of PEDV infection and transmission. Thus, an effective mucosal immune response requires secretion of both serum IgG and mucosal SIgA. Our data showed that a strong and long-lasting anti-PEDV IgG response was detected in mice orally immunized with pPG-COE-DCpep/L393 and that the anti-PEDV IgG response increased rapidly following a booster immunization. Although the SIgA antibody represents the major humoral mechanism of defense of mucosal surfaces, serum-derived IgG can also contribute significantly to immune defense, by reducing the ability of pathogens to cross the intestinal mucosa and the systemic spread of invasive pathogens, and thereby supplements the mucosal protection provided by SigA $[7,39]$. Compared with systemic immunity, the distinctive hallmark of mucosal immunity is the local production of SIgA, which can prevent binding of pathogens to the intestinal epithelial cells and remove pathogens crossing the gastric mucosal barrier [40]. Significantly, the critical advantage of the lactic acid bacteria vaccine is the ability to induce antigen-specific IgA responses in the intestinal mucosa. In this study, we observed here that oral administration of strain pPG-COE-DCpep/L393 expressing a DC-targeting peptide fused with PEDV COE antigen induced substantially elevated levels of anti-PEDV specific SIgA response in the feces, genital tract secretions, and intestinal mucus samples.Ourresults showed that oral administration of strains pPG-COE-DCpep/L393 induced a higher response than did strains pPG-COE/L393 expressing COE antigen only. However, despite statistical significance, the difference is not very large compared to the difference between a positive response and negative controls. On one hand, it may be relevant in mice species, so it need to further 
studies to show relevance in other species. On the other hand, in this study, the efficient antigen-specific immune responses of PEDV only induced by COE antigen, the presence of DCpep further potentiated the effect of recombinant COE antigen.

Extracellular cytokine analysis is a key method for evaluating the ability of a vaccine to elicit $\mathrm{Th} 1$ or Th2 responses [23]. Cytokines produced by $\mathrm{CD} 4^{+} \mathrm{T}$ helper (Th) lymphocytes could regulate immune system functions, including antibody production and cellular immune responses. The Thl cell response is closely related to cellular immune responses, whereas the Th2 cell response is associated with humoral immunity. The IL-4/IFN- $\gamma$ ratio is used to indicate the Th1 or Th2 bias of the generated immune response [41]. In this study, we determined the levels of IFN- $\gamma$ and IL-4 in the immunized mice, and observed that oral immunization with pPG-COE-DCpep/L393 can significantly elicit IFN- $\gamma$ and IL-4 production in a ratio of about 1:1, which indicates that PPG-COE-DCpep/L393 can induce the development of both Thl- and Th2-type immune responses in vivo, similar to the previous finding that use of the DCpep ligand supported both Thl- and Th2-type immune responses [23]. Recently, induction of SIgA against microbes and foreign immunogens was demonstrated to be a T cell-dependent immune response [42]. In this work, the fusion protein COE-DCpep delivered by L. casei canactivate DC, then elicit both Thl- and Th2-type immune responses, indicating that efficient $\mathrm{T}$ cell-mediated immunity was critical for SIgA induction. Moreover, pPG-COE-DCpep/L393 can also elicit a more efficient and robust lymphocyte proliferation response against PEDV COE antigen.

The translational value of studies in mice to the pig species is significant. Many vaccines regarding piglet diarrhea such as PEDV and TGEV were developed in the mice model [21,43-45]. Jiang et al. showed that oral L.casei vaccine against TGEV in mice could increase the Treg population [22]. To further evaluate the immune effect of the vaccine in the target animal, Jiang et al, using piglets as models, suggested that the oral L. casei vaccine elicit effective mucosal and systemic immune responses against TGEV infection [46]. We believe that this translational value of the oral L. casei vaccine against TGEV from mice to pig has already provided promising results, especially for the congeneric PEDV which also causes pig diarrhea. Furthermore, DCpep recognizes human, avian, canine, equine, feline and mouse's DCs [10]. So we hypothesize DCpep also bound to pig DCs due to the similarity of the immune system between pig and human. Although mice are not susceptible to PEDV infection, good immune responses of the L.casei vaccine in mice were observed in this study. The results of our study at least demonstrate that $L$. casei surface-displayed PEDV core neutralizing epitope (COE) fused with DC-targeting peptide (DCpep) could act as a novel mucosal vaccine formulation and provide a useful strategy to induce efficient immune responses against PEDV infection. Certainly, further investigations are indispensable to evaluate the immunogenicity and host immunity protection of this vaccine following its oral administration in piglets.

In conclusion, an intestinal DC-targeting oral vaccine strategy using Lactobacillus casei to deliver the core neutralizing epitope (COE) antigen of PEDV conjugated with a DC-targeting peptide as an immune adjuvant was explored to develop an anti-PEDV vaccine for oral administration in this study. We demonstrated that the genetically engineered pPG-COE-DCpep/L393 can efficiently induce mucosal, humoral, and cellular immune responses against PEDV, suggesting a promising vaccine strategy.

Acknowledgments: This work was supported by the National Key Research and Development Program of China (2016YFD0500100), the National Natural Science Foundation of China (31672591, 31472226), and the National Science and Technology Support Program in Rural Areas of the 12th Five-Year Plan (grant 2015BAD12B02-7).

Author Contributions: Yijing $\mathrm{Li}$ and Yigang $\mathrm{Xu}$ conceived and designed the study. Xiaona Wang, Li Wang, Xuewei Huang, Sunting Ma, Meiling Yu, Wen Shi performed the experiments. Xinyuan Qiao and Lijie Tang analyzed and interpreted the data. Xiaona Wang wrote the paper. All authors read and approved the manuscript.

Conflicts of Interest: The authors declare no conflict of interest. 


\section{References}

1. Pensaert, M.B.; de Bouck, P. A new coronavirus-like particle associated with diarrhea in swine. Arch. Virol. 1978, 58, 243-247. [CrossRef] [PubMed]

2. Crawford, K.; Lager, K.M.; Kulshreshtha, V.; Miller, L.C.; Faaberg, K.S. Status of vaccines for porcine epidemic diarrhea virus in the United States and Canada. Virus Res. 2016, 226, 108-116. [CrossRef] [PubMed]

3. Shima, H.; Watanabe, T.; Fukuda, S.; Fukuoka, S.; Ohara, O.; Ohno, H. A novel mucosal vaccine targeting Peyer's patch M cells induces protective antigen-specific IgA responses. Int. Immunol. 2014, 26, 619-625. [CrossRef] [PubMed]

4. Kim, S.H.; Jang, Y.S. Antigen targeting to $\mathrm{M}$ cells for enhancing the efficacy of mucosal vaccines. Exp. Mol. Med. 2014, 46, e85. [CrossRef] [PubMed]

5. Ho, P.S.; Kwang, J.; Lee, Y.K. Intragastric administration of Lactobacillus casei expressing transmissible gastroentritis coronavirus spike glycoprotein induced specific antibody production. Vaccine 2005, 23, 1335-1342. [CrossRef] [PubMed]

6. Huang, L.L.; Qin, T.; Yin, Y.Y.; Gao, X.; Lin, J.; Yang, Q.; Yu, Q.H. Bacillus amyloliquefaciens SQR9 induces dendritic cell maturation and enhances the immune response against inactivated avian influenza virus. Sci. Rep. 2016, 6. [CrossRef] [PubMed]

7. Wells, J.M.; Mercenier, A. Mucosal delivery of therapeutic and prophylactic molecules using lactic acid bacteria. Nat. Rev. Microbiol. 2008, 6, 349-362. [CrossRef] [PubMed]

8. Macpherson, A.J.; Köller, Y.; McCoy, K.D. The bilateral responsiveness between intestinal microbes and IgA. Trends Immunol. 2015, 36, 460-470. [CrossRef] [PubMed]

9. Yu, M.L.; Qi, R.M.; Chen, C.Y.; Yin, J.Y.; Ma, S.T.; Shi, W.; Wu, Y.; Ge, J.W.; Jiang, Y.P.; Tang, L.J.; et al. Immunogenicity of recombinant Lactobacillus casei-expressing F4 (K88) fimbrial adhesin FaeG in conjunction with a heat-labile enterotoxin A (LTAK63) and heat-labile enterotoxin B (LTB) of enterotoxigenic Escherichia coli as an oral adjuvant in mice. J. Appl. Microbiol. 2017, 122, 506-515. [CrossRef] [PubMed]

10. Owen, J.L.; Sahay, B.; Mohamadzadeh, M. New generation of oral mucosal vaccines targeting dendritic cells. Curr. Opin. Chem. Biol. 2013, 17, 918-924. [CrossRef] [PubMed]

11. Harman, A.N.; Kim, M.; Nasr, N.; Sandgren, K.J.; Cameron, P.U. Tissue dendritic cells as portals for HIV entry. Rev. Med. Virol. 2013, 23, 319-333. [CrossRef] [PubMed]

12. Chang, S.Y.; Ko, H.J.; Kweon, M.N. Mucosal dendritic cells shape mucosal immunity. Exp. Mol. Med. 2014, 46, e84. [CrossRef] [PubMed]

13. Mohamadzadeh, M.; Duong, T.; Sandwick, S.J.; Hoover, T.; Klaenhammer, T.R. Dendritic cell targeting of Bacillus anthracis protective antigen expressed by Lactobacillus acidophilus protects mice from lethal challenge. Proc. Natl. Acad. Sci. USA 2009, 106, 4331-4336. [CrossRef] [PubMed]

14. Wells, J. Mucosal vaccination and therapy with genetically modified lactic acid bacteria. Annu. Rev. Food Sci. Technol. 2011, 2, 423-445. [CrossRef] [PubMed]

15. De Haan, A.; Renegar, K.B.; Small, P.A., Jr.; Wilschut, J. Induction of a secretory IgA response in the murine female urogenital tract by immunization of the lungs with liposome-supplemented viral subunit antigen. Vaccine 1995, 13, 613-616. [CrossRef]

16. Xia, W.Y.; Kirkman, R.L. Immune function in transplanted small intestine. Total secretory IgA production and response against cholera toxin. Transplantation 1990, 49, 277-280. [CrossRef] [PubMed]

17. Wicht, O.; Li, W.; Willems, L.; Meuleman, T.J.; Wubbolts, R.W.; van Kuppeveld, F.J.; Rottier, P.J.; Bosch, B.J. Proteolytic activation of the porcine epidemic diarrhea coronavirus spike fusion protein by trypsin in cell culture. J. Virol. 2014, 88, 7952-7961. [CrossRef] [PubMed]

18. Makadiya, N.; Brownlie, R.; van den Hurk, J.; Berube, N.; Allan, B.; Gerdts, V.; Zakhartchouk, A. S1 domain of the porcine epidemic diarrhea virus spike protein as a vaccine antigen. Virol. J. 2016, 13, 57. [CrossRef] [PubMed]

19. Chang, S.H.; Bae, J.L.; Kang, T.J.; Kim, J.; Chung, G.H.; Lim, C.W.; Laude, H.; Yang, M.S.; Jang, Y.S. Identification of the epitope region capable of inducing neutralizing antibodies against the porcine epidemic diarrhea virus. Mol. Cells 2002, 14, 295-299. [PubMed]

20. Huy, N.-X.; Yang, M.-S.; Kim, T.-G. Expression of a cholera toxin B subunit-neutralizing epitope of the porcine epidemic diarrhea virus fusion gene in transgenic lettuce (Lactuca sativa L.). Mol. Biotechnol. 2011, 48, 201-209. [CrossRef] [PubMed] 
21. Ge, J.W.; Liu, D.Q.; Li, Y.J. Construction of recombinant lactobacilli expressing the core neutralizing epitope (COE) of porcine epidemic diarrhea virus and a fusion protein consisting of COE and Escherichia coli heat-labile enterotoxin $\mathrm{B}$, and comparison of the immune responses by orogastric immunization. Can. J. Microbiol. 2012, 58, 1258-1267. [PubMed]

22. Jiang, X.P.; Yu, M.L.; Qiao, X.Y.; Liu, M.; Tang, L.J.; Jiang, Y.P.; Cui, W.; Li, Y.J. Up-regulation of MDP and tuftsin gene expression in Th1 and Th17 cells as an adjuvant for an oral Lactobacillus casei vaccine against anti-transmissible gastroenteritis virus. Appl. Microbiol. Biotechnol. 2014, 98, 8301-8312. [CrossRef] [PubMed]

23. Shi, S.H.; Yang, W.T.; Yang, G.L.; Zhang, X.K.; Liu, Y.Y.; Zhang, L.J.; Ye, L.P.; Hu, J.T.; Xing, X.; Qi, C.; et al. Lactobacillus plantarum vaccine vector expressing hemagglutinin provides protection against H9N2 challenge infection. Virus Res. 2016, 211, 46-57. [CrossRef] [PubMed]

24. Song, B.F.; Ju, L.Z.; Li, Y.J.; Tang, L.J. Chromosomal insertions in the Lactobacillus casei upp gene that are useful for vaccine expression. Appl. Environ. Microbiol. 2014, 80, 3321-3326. [CrossRef] [PubMed]

25. Robinson, K.; Chamberlain, L.M.; Schofield, K.M.; Wells, J.M.; le Page, R.W. Oral vaccination of mice against tetanus with recombinant Lactococcus lactis. Nat. Biotechnol. 1997, 15, 653-657. [CrossRef] [PubMed]

26. Wadolkowski, E.A.; Burris, J.A.; O’brienl, A.D. Mouse model for colonization and disease caused by enterohemorrhagic Escherichia coli O157: H7. Infect. Immun. 1990, 58, 2438-2445. [PubMed]

27. Shi, S.H.; Yang, W.T.; Yang, G.L.; Cong, Y.L.; Huang, H.B.; Wang, Q.; Cai, R.P.; Ye, L.P.; Hu, J.T.; Zhou, J.Y.; et al. Immunoprotection against influenza virus $\mathrm{H} 9 \mathrm{~N} 2$ by the oral administration of recombinant Lactobacillus plantarumNC8 expressing hemagglutinin in BALB/c mice. Virology 2014, 464, 166-176. [CrossRef] [PubMed]

28. Wei, C.H.; Liu, J.K.; Hou, X.L.; Yu, L.Y.; Lee, J.S.; Kim, C.J. Immunogenicity and protective efficacy of orally or intranasally administered recombinant Lactobacillus casei expressing ETEC K99. Vaccine 2010, 28, 4113-4118. [CrossRef] [PubMed]

29. Onate, A.A.; Cespedes, S.; Cabrera, A.; Rivers, R.; Gonzalez, A.; Munoz, C.; Folch, H.; Andrews, E. A DNA vaccine encoding $\mathrm{Cu}, \mathrm{Zn}$ superoxide dismutase of Brucella abortus induces protective immunity in BALB/C Mice. Infect. Immun. 2003, 71, 4857-4861. [CrossRef] [PubMed]

30. Chen, Q.; Li, G.; Stasko, J.; Thomas, J.T.; Stensland, W.R.; Pillatzki, A.E.; Gauger, P.C.; Schwartz, K.J.; Madson, D.; Yoon, K.J.; et al. Isolation and characterization of porcine epidemic diarrhea viruses associated with the 2013 disease outbreak among swine in the United States. J. Clin. Microbiol. 2014, 52, 234-243. [CrossRef] [PubMed]

31. Lee, S.; Lee, C. Outbreak-related porcine epidemic diarrhea virus strains similar to US strains, South Korea, 2013. Infect. Dis. 2014, 20, 1223-1226. [CrossRef] [PubMed]

32. Song, D.; Moon, H.; Kang, B. Porcine epidemic diarrhea: A review of current epidemiology and available vaccines. Clin. Exp. Vaccine Res. 2015, 4, 166-176. [CrossRef] [PubMed]

33. Sun, D.B.; Wang, X.Y.; Wei, S.; Chen, J.F.; Feng, L. Epidemiology and vaccine of porcine epidemic diarrhea virus in China: A mini-review. J. Vet. Med. Sci. 2016, 78, 355-363. [CrossRef] [PubMed]

34. McGhee, J.R.; Mestecky, J. In defense of mucosal surfaces. Development of novel vaccines for IgA responses protective at the portals of entry of microbial pathogens. Infect. Dis. Clin. N. Am. 1990, 4, 315-341.

35. Zhang, N.; Hou, X.; Yu, L.; Wang, G.; Zhao, Z.; Gao, Y. Colonization and distribution of recombinant Lactobacillus casei with green fluorescent protein in mice intestine. Wei Sheng Wu Xue Bao 2010, 50, 1232-1238. [PubMed]

36. Xu, Y.G.; Cui, L.C.; Tian, C.Y.; Zhang, G.C.; Huo, G.C.; Tang, L.J.; Li, Y.J. Immunogenicity of recombinant classic swine fever virus CD8(+) T lymphocyte epitope and porcine parvovirus VP2 antigen coexpressed by Lactobacillus casei in swine via oral vaccination. Clin. Vaccine Immunol. 2011, 18, 1979-1986. [CrossRef] [PubMed]

37. Jiang, Y.L.; Hu, J.T.; Guo, Y.B.; Yang, W.T.; Ye, L.P.; Shi, C.W.; Liu, Y.Y.; Yang, G.L.; Wang, C.F. Construction and immunological evaluation of recombinant Lactobacillus plantarum expressing $\mathrm{HN}$ of Newcastle disease virus and DC- targeting peptide fusion protein. J. Biotechnol. 2015, 216, 82-89. [CrossRef] [PubMed]

38. Narita, J.; Okano, K.; Kitao, T.; Ishida, S.; Sewaki, T.; Sung, M.H.; Fukuda, H.; Kondo, A. Display of $\alpha$-amylase on the surface of Lactobacillus casei cells by use of the PgsA anchor protein, and production of lactic acid from starch. Appl. Environ. Microbiol. 2006, 72, 269-275. [CrossRef] [PubMed]

39. Holmgren, J.; Czerkinsky, C. Mucosal immunity and vaccines. Nat. Med. 2005, 11 (Suppl. S4), S45-S53. [CrossRef] [PubMed] 
40. Sutherland, D.B.; Fagarasan, S. IgA synthesis: A form of functional immune adaptation extending beyond gut. Curr. Opin. Immunol. 2012, 24, 261-268. [CrossRef] [PubMed]

41. Nan, C.L.; Lei, Z.L.; Zhao, Z.J.; Shi, L.H.; Ouyang, Y.C.; Song, X.F.; Sun, Q.Y.; Chen, D.Y. Increased Th1/Th2 (IFN- $\gamma /$ IL-4) Cytokine mRNA ratio of rat embryos in the pregnant mouse uterus. J. Reprod. Dev. 2007, 53, 219-228. [CrossRef] [PubMed]

42. Fagarasan, S.; Kawamoto, S.; Kanagawa, O.; Suzuki, K. Adaptive immune regulation in the gut: T cell-dependent and T cell-independent IgA synthesis. Annu. Rev. Immunol. 2010, 28, 243-273. [CrossRef] [PubMed]

43. Yu, M.L.; Wang, L.; Ma, S.T.; Wang, X.N.; Wang, Y.S.; Xiao, Y.; Jiang, Y.P.; Qiao, X.Y.; Tang, L.J.; Xu, Y.G.; et al. Immunogenicity of eGFP-Marked Recombinant Lactobacillus casei against Transmissible Gastroenteritis Virus and Porcine Epidemic Diarrhea Virus. Viruses 2017, 9, 274. [CrossRef] [PubMed]

44. Piao, D.C.; Shin, D.W.; Kim, I.S.; Li, H.S.; Oh, S.H.; Singh, B.; Maharjan, S.; Lee, Y.S.; Bok, J.D.; Cho, C.S.; et al. Trigger factor assisted soluble expression of recombinant spike protein of porcine epidemic diarrhea virus in Escherichia coli. BMC Biotechnol. 2016, 16, 39. [CrossRef] [PubMed]

45. Huy, N.X.; Kim, S.H.; Yang, M.S.; Kim, T.G. Immunogenicity of a neutralizing epitope from porcine epidemic diarrhea virus: $\mathrm{M}$ cell targeting ligand fusion protein expressed in transgenic rice calli. Plant Cell Rep. 2012, 31, 1933-1942. [CrossRef] [PubMed]

46. Jiang, X.P.; Hou, X.Y.; Tang, L.J.; Jiang, Y.P.; Ma, G.P.; Li, Y.J. A phase trial of the oral Lactobacillus casei vaccine polarizes Th2 cell immunity against transmissible gastroenteritis coronavirus infection. Appl. Microbiol. Biotechnol. 2016, 100, 7457-7469. [CrossRef] [PubMed]

(C) 2017 by the authors. Licensee MDPI, Basel, Switzerland. This article is an open access article distributed under the terms and conditions of the Creative Commons Attribution (CC BY) license (http:/ / creativecommons.org/licenses/by/4.0/). 